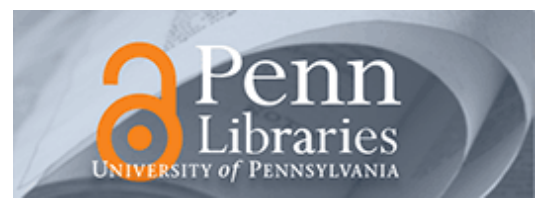

University of Pennsylvania

ScholarlyCommons

\title{
6-1988
}

\section{Strict Global Lyapunov Functions for Mechanical Systems}

\author{
Daniel E. Koditschek \\ University of Pennsylvania, kod@seas.upenn.edu
}

Follow this and additional works at: https://repository.upenn.edu/ese_papers

Part of the Electrical and Computer Engineering Commons, and the Systems Engineering Commons

\section{Recommended Citation}

Daniel E. Koditschek, "Strict Global Lyapunov Functions for Mechanical Systems", Proceedings of the 1988 American Control Conference, 1770-1775. June 1988.

Copyright 1988 IEEE. Reprinted from Proceedings of the 1988 American Control Conference, pages 1770-1775. This material is posted here with permission of the IEEE. Such permission of the IEEE does not in any way imply IEEE endorsement of any of the University of Pennsylvania's products or services. Internal or personal use of this material is permitted. However, permission to reprint/republish this material for advertising or promotional purposes or for creating new collective works for resale or redistribution must be obtained from the IEEE by writing to pubs-permissions@ieee.org. By choosing to view this document, you agree to all provisions of the copyright laws protecting it.

NOTE: At the time of publication, author Daniel Koditschek was affiliated with Yale University. Currently, he is a faculty member in the Department of Electrical and Systems Engineering at the University of Pennsylvania.

This paper is posted at ScholarlyCommons. https://repository.upenn.edu/ese_papers/679

For more information, please contact repository@pobox.upenn.edu. 


\title{
Strict Global Lyapunov Functions for Mechanical Systems
}

\author{
Abstract \\ A novel Lyapunov function is introduced. The author treats the PD compensated mechanical system, \\ which is defined on a configuration space which admits a trivial tangent bundle. Previous results are \\ derived in a simpler form, namely, that such systems are exponentially stable, hence BIBO stable, and \\ steady-state ouptut magnitudes are proportional to input magnitudes with a constant of proportionality \\ depending in a simple fashion on the PD gain magnitudes. \\ For more information: Kod*Lab \\ Disciplines \\ Electrical and Computer Engineering | Engineering | Systems Engineering \\ Comments \\ Copyright 1988 IEEE. Reprinted from Proceedings of the 1988 American Control Conference, pages \\ 1770-1775. \\ This material is posted here with permission of the IEEE. Such permission of the IEEE does not in any way \\ imply IEEE endorsement of any of the University of Pennsylvania's products or services. Internal or \\ personal use of this material is permitted. However, permission to reprint/republish this material for \\ advertising or promotional purposes or for creating new collective works for resale or redistribution must \\ be obtained from the IEEE by writing to pubs-permissions@ieee.org. By choosing to view this document, \\ you agree to all provisions of the copyright laws protecting it. \\ NOTE: At the time of publication, author Daniel Koditschek was affiliated \\ with Yale University. Currently, he is a faculty member in the Department of \\ Electrical and Systems Engineering at the University of Pennsylvania.
}




\title{
Strict Global Lyapunov Functions for Mechanical Systems
}

\author{
Daniel E. Koditschek • \\ Center for Systems Science \\ Yale University, Department of Electrical Engineering
}

\begin{abstract}
This paper presents a strict, global Lyapunov function for the class of dissipative mechanical systems defined on a configuration space which admits a trivial tangent bundle.
\end{abstract}

\section{Introduction}

The "mechanical systems" define a large and important class of highly nonlinear dynamical equations. For example, all robot dynamics are are modeled by such systems. Since there can be no "operational calculus", Lyapunov theory offers an attrac. tive alternative approach to a constructive control methodology for this class of systems: it is not hard to translate many frequency domain insights into this language in the linear case. [8]. Generalizing such an approach obviously depends upon the availability of "adequate" Lyapunov functions.

Here it seems on first impression as though nature has been kind. There is a "canonical" Lyapunov function for dissipative mechanical systems - the total energy - as has been known for since the nineteenth century. This scalar valued map enjoys the property of global defnition, since it is con. structed in terms of the intrinsic Riemannian geometry of the configuration space. Unfortunately, it results in a semi-definite derivative along trajectories of a mechanical system, and the full strength of Lyapunov's method is lost.

Recently, Arimoto [2], Bayard and Wen [15], and the present author $[5,6]$ have, all independently, devised strict Lyapunov functions for "PD" compensated mechanical systems. All three constructions are remarkably similar, and share the common fault of achieving only a locally negative definite derivative (the local domain may be arbitrarily enlarged) on the phase space. The central contribution of this paper, Theorem 1, is the construction of a new Lyapunov function for the dissipative mechanical systems which is global - defined in terms of the intrinsic Riemannian geometry of an arbitrary mechanical system - and strict - the derivative is negative definite on the phase space of (tangent bundle over) any parallelizable configuration space, vanishing only on the equilibrium states of the vector field. 1

This paper, being of an essentially analytical nature, will make only illustrative use of the new construction to re-derive (hopefully in a simpler form) the useful results concerning $P D$ compensated (quadratic potential) mechanical systems on the "Euclidean $n$-disk" reported in the conference papers cited previously $[2,15,6]$. Namely, such systems are exponentially stable, hence BIBO stable, and steady state output magnitudes may be shown to be proportional to input magnitudes with a constant of proportionality depending in a simple fashion upon the PD gain magnitudes. The larger motivation for this work is the hope of applying the construction to more general robotic problems where the configuration space is not homeomorphic to a disk and the potential energy is not quadratic. This work is currently in preparation.

'The limitation to parallelizable spaces - i.e., thase whose tangens bundle is trivial - seems to be an artifact of the present proof rather than on intrinsic limitation in the construction. This issue is presently under investigation.
The next section presents the ingredients leading to the standard definition of a dissipative mechanical system on a smooth configuration space. Section 3 introduces the new Lyapunov function. Finally, Section 4 treats the PD compensated mechanical system, as an example of the construction in the familiar case. A variety of technical results and computations are not included in this paper for lack of space - the reader is referred to the background technical report [4] for further details.

\section{Dissipative Mechanical Systems}

The geometry of classical physics has been extensively studied for decades, and recent years have witnessed the publication of numerous expository texts containing the background ma. terial required for the present paper. This section sketches the relevant ideas, appealing frequently to the excellent text of Abraham and Marsden [1].

\subsection{Notation}

The tangent bundle over a manifold, $J$, will be denoted $T J$ with projection map $r: T J \rightarrow J$. The coordinate representation of a map will be denoted by underlining. For the purposes of this paper, the manifold, $J$, will be a simply connected open subset of $\mathbb{I R}^{n}$, which admits a single global chart, and, when there is no chance of confusion, coordinate representations of maps will often be denoted by the same symbol as the original map. Taking the jacobian of a map between Euclidean Vector spaces is denoted by $D$, in contrast to taking the differential one form of a scalar valued map on an arbitrary manifold, $d$.

Following Hirsch $\{3\}$, if $x, y$ are two vector bundles over $J$, then define the morphisms between them, $\mathcal{M r}^{r}[X, y]$, to be the set of all smooth (i.e. continuously differentiable $r$ times) fiber preserving maps which are linear on each fiber, i.e.,

$$
\begin{aligned}
& M^{r}[x, y\} \triangleq\left\{M \in C^{r}[x, y]:\right. \\
& \left.M \mid \pi \bar{x}^{-1}(q) \in \mathcal{L}\left(\pi \bar{x}^{-1}(q), \pi_{y}^{-1}(q)\right) \text { for all } q \in J\right\} \text {. }
\end{aligned}
$$

Transpose, symmetric, and positive definite morphisms are presented in [4], along with the notion of upper and lower magnitude functions, and the group of scalar comparison func.
tions,

$$
\begin{aligned}
& K_{r} \triangleq\left\{\kappa \in C^{r}\left[\mathbb{R}^{+}, \mathbb{R}^{+}\right]:\right. \\
& \quad \lim _{t \rightarrow 0} \kappa(t)=0 \text { and } \lim _{t \rightarrow \infty} \kappa(t)=\infty \\
& \text { and } \left.t_{1}<t_{2} \Rightarrow \kappa\left(t_{1}\right)<\kappa\left(t_{2}\right)\right\},
\end{aligned}
$$

which are used to define Lyapunov functions on manifolds.

\subsection{Mechanical Systems}

\subsubsection{Kinetic Energy as a Riemannian Metric}

Define a mechanical system to be the Lagrangian dynamical system resulting from a cost functional specified by a Rie-

\footnotetext{
1This work is supported in part by the National Science Foundaton under grant no. DMC.8505160
} 
mannian metric - a positive definite symmetric morphism $M \in M^{\omega}\left\{T J, T^{*} J\right\}$, as discussed in $[4]$ - which specifies an inner product $\langle\cdot \mid \cdot\rangle$ on $\left.T_{q}\right\rangle$ for every point of configuration spacc, $q \in J$. The author has presented a a quick derivation of the Riemannian metric resulting from the sort of physical system which motivates the paper - the kinetic energy ${ }^{2}$, $\kappa(v) \triangleq \frac{1}{2}\langle v \mid v\rangle$ of a "kinematic chain" encountered in robotics - in a recent encyclopedia article [8].

A Riemannian metric induces an affine connection, $\nabla$, with respect to which parallel translation preserves the metric inner product [1].This implies that for any two curves, $c_{1}, c_{2}$, in $T J$ over the same base curve, $b \equiv \tau \circ c_{1} \equiv \tau \circ c_{2}$, we have

$$
\frac{d}{d t}\left\langle c_{1} \mid c_{2}\right\rangle=\left\langle\nabla_{b} c_{1} \mid c_{2}\right\rangle+\left\langle c_{1} \mid \nabla_{b} c_{2}\right\rangle
$$

taking the covariant derivative, along the curves $c_{i}$, consid. cred as vector fields over the base curve, $b[9]$.

\subsubsection{Coordinate Representation of the Lagrangian Vector Fleld}

Each point, $q \in J$, admits an open neighborhood, $0 \subseteq J$, on which the "local phase space at $q$ ", is a Euclidean vector space. Thus, a point, $v \in T J$ admits a local coordinate representation,

$$
p \triangleq\left[\begin{array}{l}
p_{1} \\
p_{2}
\end{array}\right] \in \mathbb{R}^{2 n} .
$$

For the purposes of the present paper, the configuration space may be presumed a subset of $\mathbb{R}^{n}$, thus, $T J$ is trivial - i.e. it is a subset of $\mathbb{R}^{n} \times \mathbb{R}^{n}$ - and this local coordinate system is valid on the entire phase space, $T J$.

The equations of motion in the local coordinates resulting from application of the "Euler-Lagrange" operator, to the kinetic energy, $\kappa$, take the form

$$
\ddot{p_{1}}+\Gamma_{p_{1}}\left(\dot{p}_{1}, \dot{p_{1}}\right)=0
$$

where $\Gamma$ represents the Christoffel symbols for the given coor. dinates. The relation of $\mathrm{P}$ to the coordinate expression of the morphism, $M$, is derived in [4] as

$$
\begin{aligned}
\Gamma_{p_{1}}(x, y) & =\frac{1}{2} M^{-1}\left(p_{1}\right) C\left(p_{1}, x\right) y \\
C\left(p_{1}, x\right) y & \triangleq\left[\underline{M}_{p_{1}}(x) y+\dot{M}_{p_{1}}(y) x-\underline{M}_{p_{1}}(x)^{\mathrm{T}} y\right] \\
\underline{M}_{p_{1}}(x) & \triangleq(x \otimes I)^{\mathrm{T}} D \underline{M}^{\mathrm{s}},
\end{aligned}
$$

where it is also shown that $\Gamma$ is symmetric $-\Gamma(x, y)=\Gamma(y, x)$ - so that this is the expression of the unique torsion free metric connection. 3 This results in the local representation of $f_{x}$ as

$$
\begin{aligned}
& \dot{p_{1}}=p_{2} \\
& \dot{p_{2}}=-M^{-1}\left(p_{1}\right) C\left(p_{1}, p_{2}\right) p_{2} .
\end{aligned}
$$

While $T J$ is endowed with a natural metric induced by the Riemannian metric, $M$, as described in [4], the present paper will require only a local notion of distance, for purposes of applying comparison functions to $T J$ [4]. In such applications

\footnotetext{
${ }^{3}$ Kinetic Energy is formally defined as a scalar valued map on $T^{*} \mathcal{J}$ (1)|Def. 4.5.2), howeyer it will ease the discussion and do no technical harm in the present paper to apeat of $x a \mathrm{amap}$ on $T J$.

In earlier papers, $[7,6]$, the author bas nawittinaly ued the local expression for a metric connection with non-zero torsion. While the geodesics of two connections which differ only by tosion are identical, the non-zero torsion necessitates annoying additional cancellation of "defect" terms in control applications. It is worth pointing out that other authors have independently made use of the preferable zerotorsion connection in their control applications $\{15,12\}$.
}

it suffices to use the Euclidean norm topology induced by the metric of the local coordinate system. A metric, $\rho$, having been chosen, any morphism, $F$, gives rise to scalar valued maps on $J, \nu_{F}(q), \mu_{F}(q)$, as introduced in [4], which measure its smallest and greatest "magnitudes" on each fiber, and lead to an obvious construction of a quadratic comparison function on each fiber.

Lemma 1 For every $q \in J$, the kinetic energy, $x$, is a nondegenerate positive definite function on $T_{q} J$, possessed of a quadratic lower comparison function, in coordinates,

$$
\kappa\left(p_{1}, p_{2}\right) \geq \overline{\nu_{M}}\left\|p_{2}\right\|^{2}
$$

\subsection{Gradient Vector Flelds in Mechanical Sys* tems}

\subsubsection{Morse Functions and Their Gradient Vector Flelds}

Consider the class of twice differentiable real valued functions $\varphi \in C^{2}[J, \mathbb{R}]$. The co-vector field, $d \varphi$, is related to the gradient vector field, grad $\varphi$, of $\varphi$ by the identity

$$
\text { grod } \rho \triangleq M^{-1} d \varphi \text {. }
$$

Note that the "hessian" of the original function is obtained from the jacobian, $\bar{K}_{1} \triangleq d \varphi_{\text {, }}$, in local coordinates,

$$
\tilde{K}_{1}\left(p_{1}\right) \equiv\left[D^{2} \underline{\underline{\rho}}\right]\left(p_{1}\right) .
$$

One calls $\varphi$ a Morse function if its hessian is non-singular at every critical point [3].

\subsubsection{Lifting Gradient Vector Fields}

Any smooth real valued function, $\varphi: J \rightarrow \mathbb{R}$ may be "pulled back" to $T J$ in a natural fashion by defining $\tilde{\varphi} \triangleq \varphi \circ T$. This defines a new Lagrangian function,

$$
\lambda \triangleq \kappa-\ddot{\varphi}
$$

whose Lagrangian vector field, $f_{x-\check{\varphi}}$, can be shown to include a "lift" of the gradient vector field of $\varphi$ in the following sense. It can be shown [1] that $c$ is a trajectory of $f_{x-p}$ if and only if its base integral curve, $b \triangleq \tau o c$ has the property

$$
\nabla_{\dot{b}} \dot{b}=-\operatorname{grad} \varphi \circ b \text {. }
$$

This implies that the resulting Lagrangian vector field may be written as

$$
f_{\kappa-\phi}(x)=f_{\kappa}(x)-V_{z}(\operatorname{grad} \varphi) \circ \tau(x),
$$

where $V$ is the vertical lift.

\subsubsection{Coordinate Representation of a Mechanical Sys- tom with Good Potentlal Energy}

Say that $\varphi \in C^{2}\left[J, \overline{\mathbb{R}^{+}} \mid\right.$is a good potential function at $q_{0}$ if it is a Morse function on $J$, positive definite at $q_{0}$, and bounded away from zero everywhere else on $J$. This means that there can be found a lower comparison function, $\lambda_{\varphi} \in X_{2}$, for $\varphi$, in the sense defined in [4].

$$
\lambda_{\varphi} \circ \rho,\left(q_{0}, q\right) \leq \varphi(q)
$$

In such a case, $q_{0}$ is a local minimum of $\varphi-$ an attracting equilibrium state of -grad $\varphi$. It follows that $\|d \dot{ } \varphi\|$ is a

\footnotetext{
'The metric, $M$, on $T J$ induces metric on $T^{*} J$, wh respect to which
} the norm of a co-vector is well dellned. 
decrescent positive definite function at $q_{0}$, admitting the upper comparison, $v_{d \varphi} \in K_{1}$, with the property

$$
u_{d \varphi} \circ \rho,(q 0, q) \geq\|d \varphi\| \text {. }
$$

Using the same arguments as in [3]|Lem.6.1.1], given any local coordinate system on a neighborhood, 0 , of $q \in J, \varphi$ induces a smooth map $K_{1}: \mathbb{R}^{n} \rightarrow \mathcal{L}\left(\mathbb{R}^{n}, \mathbb{R}^{n}\right)_{y}$ such that $M^{-1}\left(p_{1}\right) K_{1}\left(p_{1}\right) p_{1}$ is a representation of grad $\varphi$. It now follows that the vector field, $f_{x-\tilde{\varphi}}$ admits the coordinate representa. tion

$$
\begin{aligned}
& \dot{p_{1}}=p_{2} \\
& \dot{p_{2}}=-\underline{M}^{-1}\left(p_{1}\right)\left(C\left(p_{1}, p_{2}\right) p_{2}+K_{1}\left(p_{1}\right) p_{1}\right)
\end{aligned}
$$

\subsection{Strict Dissipative Vector Fields and Exter- nal Forces}

Following Abraham and Marsden, once again, say that a smooth vector field, $f_{d}$, on $T J$ is dissipative (1) if it is vertical and bas the property with respect to the kinetic energy, $\kappa$, that

$$
d \kappa f_{d} \leq 0 .
$$

Since / $d$ is vertical, it may be uniquely associated with a smooth fiber map, $k_{2}: T \mathcal{S} \rightarrow T^{*} \mathcal{J}$, via lifting

$$
f_{d}(v) \triangleq-V_{v} \circ M^{-1} k_{2}(v)
$$

If the product $k_{2}(v) \cdot v$ is a positive definite map on $T_{q} J$ for every $q \in J$, then say that $f_{d}$ is a strict dissipative vector field. In this case, there may be found a smooth map, $K_{2}: T_{q} J \rightarrow \mathcal{L}\left(T_{q} J, T_{q}^{*} J\right)$ such that $k_{2}(v)=K_{2}(v) v$, and $\left(K_{2}\right)_{\text {, }}$, the symmetric part of $K_{2}$ as defined in $[4]$ is always positive definite.

Lemma $2 J / f_{d}$ is a strict dissipative vector field on $T J$, then $-d \kappa \int_{d}$ is a positive definite function on $T_{q} J$ with a quadratic lower comparison function, in coordinates,

$$
k_{2}\left(p_{1}, p_{2}\right) \cdot p_{2} \geq \overline{\nu_{K_{2}}}\left\|p_{2}\right\|^{2} \text {. }
$$

\subsection{Dissipative Mechanical Systems}

To summarize, a kinetic energy law, $\kappa$, determined by a Rie. mannian metric, $M$, on $J$, induces a mechanical system (1) through the Lagrangian vector field, $f_{n}$, whose base integral curves are geodesic with respect to $M$. The addition of a potential function, $\varphi$ results in a new Lagrangian vector field,

$$
f_{x-\dot{\varphi}}=f_{x}-V(\operatorname{grad} \varphi) \circ \tau,
$$

whose integral curves conserve total energy, $\eta=\kappa+\tilde{\varphi}$. Finally, the choice of a dissipative vector field, $f_{d}$, completes the list of ingredients. We will limit attention to following class of mechanical systems:

Deflnition:

$A$ dissipative mechanical system is defined by the vector field

$$
f \triangleq f_{x-\beta}+f_{d}
$$

where

1. $N$ is the kinetic energy induced by a Riemannian metric, $M$, on $J_{;}$

2. $\varphi$ is a "good potential function" in the sense, of Section $2.9 .9 ;$

3. $\int_{d}$ is a "strict" dissipalive vector field in the.sense of Section 2.1.
In local coordinates, the disturbed dissipative mechanical system can be written,

$$
\begin{aligned}
& \dot{p_{1}}=p_{2} \\
& \dot{p_{3}}=-\underline{M}^{-1}\left(p_{1}\right)\left(\left[C\left(p_{1}, p_{2}\right)+\underline{K}_{2}\left(p_{1}, p_{2}\right) \mid p_{2}+\underline{K}_{1}\left(p_{1}\right) p_{1}\right)\right.
\end{aligned}
$$

\section{The Stability of Dissipative Mechani- cal Systems}

Lord Kelvin showed that total energy, $\eta=\kappa+\varphi$, is a Lyapunov function for a dissipative mechanical system in 1886 [14]. Arimoto and colleagues $[10,13\}$ contributed a precise demon. stration that every minimum of the potential field is a local attractor by application of LaSalle's Invariance Principle. Similar independent work of Van der Schaft [11] and this author [7] appeared subsequently. Of course, the central idea of energy dissipation is to be found in the standard texts as well, [1] Prop. 3.7.17]. Since $\eta$ is not a strict Lyapunov function that is, the Lie derivative, $L_{f}(\eta)$ is negative semi-definite conclusions about asymptotic properties of the flow near $(90,0)$ require the application of LaSalle's Invariance Principle. This limits application to autonomous dynamics, precluding any input/output analysis of the effects of the external disturbance. This flaw will now be corrected: we will "fix" Lord Kelvin's formula by adding a "cross term" to the total energy function which makes its derivative along the motions negative definite.

\subsection{The "Potentlal Angle" and Energy Scaling Function}

The concern of this section, is the construction of a new scalar valued map on $T J$ whose co-vector field acts on the portion of $f$ which is "ignored" by $d \eta$ - the "vertical lift" of the potential gradient vector field. Namely, a map, $\alpha \in C^{1}[T J, \mathbb{R}]$, is constructed to measure the "angle" between the desired ve. locity of the lifted gradient flow, and the actual velocity of the dissipative system.

The Riemannian metric is a symmetric isomorphism in $\mathcal{N}^{\omega}\left[T J, T^{\bullet} J\right]$. By choosing a morphism in the other direction one obtains the desired angular comparison, as follows. Say that a morphism, $F \in \mathcal{M}^{\omega}\left[T^{*} J, T J\right]$, is a pre-metric if its symmetric part is a "positive definite" operator on each fiber. Given a manifold, $J$, with a Riemannian metric, $M$, on the tangent bundle, $\tau: T J \rightarrow J$, a pre-metric, $F$, and a scalar valued map, $\varphi \in C^{l}[J, \mathbb{R})$, define the potential angle map, $\alpha \in C^{l}[T J, \mathbb{R}]$ by

$$
\alpha(v) \triangleq\langle F d \varphi \circ \tau(v) \mid v\rangle_{\Lambda} .
$$

Lemma 3 The Lie derivative of the potential angle map, $\alpha$, along the dissipative mechanical system, $f,(O)$, is a scalar valued map on $T J$ which may be expressed as

$L_{f} \alpha(v)=\langle B v \mid v\rangle-\left\langle F d \varphi \circ r(v) \mid k_{2}(v)\right\rangle-(d \varphi \cdot F d \varphi) \circ r(v)$.

where $B \in \mathcal{N}^{\omega}[T J, T J)$.

Proof: If $c$ is any smooth curve in $T J$, then it may considered as vector field along the base curve, $r \circ c \triangleq b$. Thus,

$$
\frac{d}{d t} \alpha \circ c=\left\langle\nabla_{j} F d \varphi \circ b \mid c\right\rangle+\left\langle F d \varphi \circ b \mid \nabla_{b} c\right\rangle
$$


since $\nabla$ is the Riemannian connection. If $c$ is the trajectory of any second order system then

$$
\dot{b}=T_{T} \circ \dot{c}=c,
$$

hence

$$
\begin{aligned}
\frac{d}{d 1} \alpha \circ c & =\left\langle\nabla_{c} F d \varphi \circ b \mid c\right\rangle+\left\langle F d \varphi \circ b \mid \nabla_{b} \dot{b}\right\rangle \\
& =\langle B c \mid c\rangle+\left\langle F d \varphi \circ b \mid \nabla_{b} b\right\rangle
\end{aligned}
$$

where $B \in \mathcal{M}^{\omega}[T J, T J]$ denotes the morphism $\nabla F d \varphi$. Since $b$ is the base integral curve of a dissipative mechan. ical system it follows that

$$
\nabla_{\dot{b}} \dot{b}=-\operatorname{grad} \varphi \circ b-k_{2}(c),
$$

hence

$$
\begin{aligned}
\frac{d}{d l} \alpha \circ c= & \langle B c \mid c\rangle-\left\langle F d \varphi \circ b \mid k_{2}(c)\right\rangle \\
& -\langle F d \varphi \circ b \mid \operatorname{grad} \varphi \circ b\rangle \\
= & \langle B c \mid c\rangle-\left\langle F d \varphi \circ b \mid k_{2}(c)\right\rangle \\
& -(d \varphi \cdot F d \varphi) \circ b,
\end{aligned}
$$

and the result follows

The pre-metric property of $F$ insures that the last term of $L_{f}(\alpha)$ is globally non-positive, vanishing only on the critical points of $\varphi$, since $F$, defines a positive definite quadratic form on the co-tangent bundle. Thus, adding $\alpha$ to $\eta$ introduces a negative term in the derivative which does not vanish automatically on the zero section of $T J$. Unfortunately, $\alpha$, itself, is sign indefinite, and its derivative along trajectories of $f$ incurs two additional sign indefinite terms as well as computed by Lemma 3 . It will now be shown that a "rescaling" of $\eta$ obtained by composition with a suitable comparison function, $\gamma \in X_{1}$, serves simultaneously to dominate the indefinite terms of both $\alpha$ and its derivative. Specifically, define

$$
\begin{aligned}
\gamma(x) \triangleq \gamma_{0} \chi & +\gamma_{1}\left(v_{d \varphi}^{2} \circ \lambda_{\varphi}^{-1}\right)(\chi) \\
& +\gamma_{2}\left[\int_{0}^{x}\left(v_{(M B)} \circ \lambda_{\eta}^{-1}\right)(\sigma) d \sigma\right],
\end{aligned}
$$

where $\gamma_{0}, \gamma_{1}, \gamma_{2}$ are positive non-decreasing scalar functions to be chosen below, and $v_{(N B)}$, is a smooth non-decreasing function which satisfies the inequality

$$
v_{(M B)},(x) \geq \sup _{,(80 . q) \leq x} \mu(M B),(q),
$$

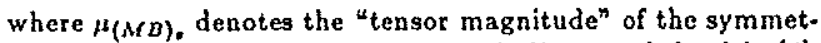
ric part of the morphism $M B$ on each fiber, as defined in [4], and $v_{d \varphi}$ is an upper comparison function for $\|d \varphi\|$ as defined in Section 2.3.3.

\subsection{Verification of the Construction}

First observe that $\gamma$ preserves the sign definite properties of $\eta$ when their composition is added to $\alpha$.

Proposition 1 For every dissipative mechanical system, $f$, (8) and potential angle map, $\alpha,(10)$, there can be found a comparison function, $\gamma \in K_{1}$, of the form (11), such that

$$
\vartheta \triangleq \gamma \circ \eta+\alpha
$$

is a positive definite function in $C^{1}\left|T J, \overline{\mathbb{R}^{+}}\right|$.
Proof: Note that $\vartheta$ vanishes at $v_{0}=\left(q_{0}, 0\right)$ as required. We first seek to show that $\theta$ is positive definite. Note that, in coordinates,

$$
\eta \geq \eta \triangleq \lambda_{\rho}\left(\left\|p_{k}\right\|\right)+\nu_{M}\left\|p_{2}\right\|^{2},
$$

according to (5). Moreover,

$$
\gamma(\chi) \geq \gamma(\chi) \triangleq \gamma_{0} \chi+\gamma_{1}\left(v_{d \varphi p}^{2} \circ \lambda_{p}^{-1}\right)(x)
$$

from the definition of $\gamma$ and (6) - it follows that

$$
\gamma \circ \eta \geq \gamma^{\prime} \eta \eta \geq \gamma_{0} \bar{\nu}_{\lambda+\gamma}\left\|p_{2}\right\|^{2}+\gamma_{1} v_{d \rho}^{2}\left(\left\|p_{1}\right\|\right) .
$$

Notice, moreover, in coordinates, that

$$
|\alpha| \leq\left|D_{\varphi}\left(p_{1}\right) E^{\mathrm{T}} \mathcal{L}_{\left(p_{2}\right.}\right| \leq v_{d \varphi}\left(\left\|p_{1}\right\|\right) \cdot \mu_{F^{\top}}\left(p_{1}\right) \cdot\left\|p_{2}\right\|_{3}
$$

where $\mu_{F} T_{M}\left(p_{1}\right)$ is again the tensor magnitude function defined in [4]. It follows that

$$
\vartheta \geq \gamma_{1} v_{d \varphi}^{2}-\mu_{F} T_{M} v_{d \varphi}\left\|p_{2}\right\|+\gamma_{0} \overline{\nu_{M K}}\left\|p_{2}\right\|^{2}
$$

and it suffices ${ }^{s}$ to find non-decreasing functions, $\gamma_{0}, \gamma_{1}$; such that $\gamma_{i} \circ \eta$ is smooth and

$$
\left[\begin{array}{cc}
\gamma_{1}(\eta) & \frac{1}{2} \mu_{F} \tau_{M}\left(p_{1}\right) \\
\frac{1}{2} \mu_{F} \tau_{M}\left(p_{1}\right) & \gamma_{0}(\eta) \nu_{M}\left(p_{1}\right)
\end{array}\right]>0 .
$$

For this, the conditions

$$
\gamma_{0} \geq 1 ; \quad \gamma_{1} \geq \frac{1}{4 \overline{\nu_{M}}} v_{F} \tau_{M} \circ \lambda_{\eta}^{-1},
$$

are sufficient, where $v_{F} T_{M}$ is a smooth non-decreasing function satisfying the inequality

The addition of the "angle measurement" in $v$ results in a locally negative definite derivative, $\dot{\theta}$. In fact, the appropriate rescaling of $\eta$ affords dominance of the negative definite terms "as globally as the potential function can allow". This is made precise by the following central result.

Theorem 1 Let $f$ be a dissipative mechanical system. Then for every valid potential angle map, $\alpha,(10)$, and a compari. son function, $\gamma \in X_{1}$, of the form (11), the positive definite function,

$$
\vartheta \triangleq \gamma \circ \eta+\alpha
$$

has a derivative along trajectories of $f$,

$$
\dot{\vartheta}=L_{f}(\vartheta)
$$

which is non-positive on $T J$ and vanishes only on the equilibrium states of $f$.

Proof: $\quad$ Let $\gamma \in X_{1}$ be chosen as in (11), so that $\vartheta$ is positive definite in consequence of Proposition 1. We have, along any curve, $c$, in $T J$,

$$
\dot{\vartheta}=\left(\gamma^{\prime} \circ \eta\right) \dot{\eta}+\dot{\alpha}
$$

If $c$ is a trajectory of $f$, then, in coordinates,

$$
\dot{\eta}=-p_{2}^{\mathrm{T}} K_{2}\left(p_{1}, p_{2}\right) p_{2}
$$

\footnotetext{
SThe sufficiency depends upon the fact that $v_{\alpha,}$ is an upper comparison function for \|dell, hence (in contrast, for instance, to a lower com. parison (unction) may be guaranteed not to vanish except at the point go.
} 
Now $\gamma^{\prime}$, the derivative of $\gamma$, satisfies

$$
\gamma^{\prime}(\chi) \geq \gamma_{0}+\gamma_{2} v_{(M B)} \circ \circ \lambda_{\eta}^{-1}(\chi),
$$

since all constituent functions of $\gamma$ are non-decreasing. From $\lambda_{\eta}^{-1} \circ \eta \geq\|p\|$, it follows that hence,

$$
v_{(M B)} \circ \circ \lambda_{\eta}^{-1} \circ \eta \geq v_{(M B)}(\|p\|) \geq v_{(M B)} .\left(\left\|p_{1}\right\|\right)
$$

$$
\underline{\dot{\theta}} \leq-p_{2}^{\tau} \underline{K}_{2} p_{2}\left(\gamma_{0}+\gamma_{2} \mu_{(\Lambda B)_{0}}\right)+\dot{\dot{\alpha}} .
$$

Using the results of Lemma 3 , expressed in coordinates, we have

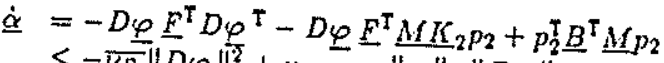

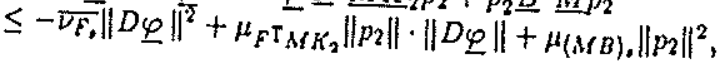

$$
\overline{\nu_{F_{r}}} \triangleq \inf _{q \in}, \nu_{F_{0}}(q)
$$

is a positive constant according to the assumption that $F$ is a pre-metric. It now follows that

$\dot{\vartheta} \leq-l(p)^{\top} Q(p) l(p)+v_{(M B)}\left(\left\|p_{1}\right\|\right)\left(1-\gamma_{2}(\eta) \overline{\sigma_{K_{2}}}\right)\left\|p_{2}\right\|^{2} ;$

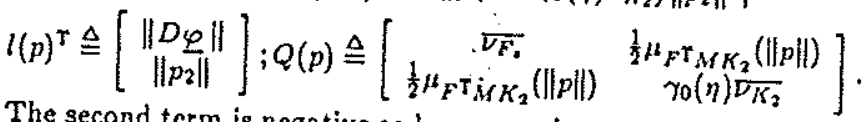
The second term is negative as long as $\gamma_{2}$ is greater than the constant function,

$$
\gamma_{2}>\frac{1}{\overline{V_{K_{2}}}}
$$

where $\overline{V_{K_{2}}}$ is specified in Lemma 2 . The $2 \times 2$ matrix is negative definite when

$$
\begin{array}{cl}
\gamma_{0} & >1+\left(\frac{1}{4 \bar{F}_{1}}\right) v_{F K_{2}}^{2} \mathrm{v}_{M K_{2}} \circ \lambda_{\eta}^{-1} \\
v_{F^{\top}{ }_{M K_{2}}}(x) & \cong \sup _{\|p\| \leq x} \sup _{\|x\|=1}\left\|E^{\top} M K_{2}\left(p_{1}, p_{2}\right) x\right\| .
\end{array}
$$

which satisfies the earlier condition (12) as well. 6 Under these conditions, $\dot{\theta}$ is negative except at the critical points of $\varphi$ as identified in the zcro section of $T J-$ the equilibrium states of $f$.

In the next section we consider the application of this construction to disturbed mechanical systems on a configuration space which is guaranteed to admit Morse functions with a sin. gle critical point - an open disk in $\mathbb{R}^{n}$. The application to nonautonomous mechanical systems on a configuration space with non-unity Euler characteristic - e.g., the punctured manifolds which result from robot obstacle avoidance problems with moving obstacles - is the subject of a separate paper.

\footnotetext{
${ }^{6}$ Note that Y $0 \eta$ is still $C^{1}$ with these definitions, following the same argument as in Proposition 1.
}

\section{Application to a PD Controlled $\mathrm{Me}$ - chanical System}

There are relatively few occasions when $J$ admits a Morse func. tion with a single critical point. In this section we examine in detail one such (practically significant) case where $J$ is a an open $n$-disk. In such a case, $\|d \varphi\|$ being radially ubounded, $\dot{\theta}$ is radially unbounded as well.

All symbols will refer to the local coordinate representation. The kinetic energy remains in the general form,

$$
\kappa(p) \triangleq p_{2}^{\top} M\left(p_{1}\right) p_{2}
$$

where $M$ is analytic in $p_{1}$, and takes values in the set of positive definite symmetric matrices. The only further restriction (imposed for computational convenience, and because it corresponds the situation in robotics) is that both $M$ and its derivatives are bounded on phase space, so that there exists an upper bound, $\overline{\mu_{M f}}$, on the the morphism norm as well as a lower bound, $\overline{D_{M}}$, and there is a linear spread estimate with constant $\sigma_{M} \triangleq \sqrt{\overline{\mu_{M}} / \overline{\nu_{M}}}$ as defined in [4].

The potential energy is defined to be that of a "perfect" Hook's law spring,

$$
\varphi\left(p_{1}\right) \triangleq p_{1}^{\top} K_{1} p_{1}
$$

where $K_{\mathrm{l}}$ is a positive definite symmetric matrix. The dissipative field is defined to be a Rayleigh field, $k_{2}(p) p_{2} \triangleq K_{2} p_{2}$, where $K_{2}$ is a positive definite symmetric matrix as well. There is an external force, as well. Note that the operator bounds on $K_{1}, K_{2}$ are all constant, according to the definitions in [4].

This corresponds to a "linear proportional and derivative feedback compensator", applied to a pure mechanical system (1), the entire closed loop subsequently "forced" by the con. trol input, $u(t)$, giving tise to the nonlinear control system, (9), where $K_{1}, K_{2}$ are constant positive definite symmetric matri-
ces.

\subsection{Construction of a Strict Lyapunov Function}

Applying the definitions from Section 3.1, the various bounding functions associated with $\varphi$ are

$$
\lambda_{\varphi}^{-1}(x)=\sqrt{\frac{\chi}{\nu_{K_{1}}}} ; \quad v_{d \varphi}(x)=\mu_{K_{1}} \chi_{i} \quad v_{d \varphi}^{2} \circ \lambda_{\varphi}^{-1}(x)=\mu_{K_{1}} \sigma_{K_{1}}^{2} \chi .
$$

The construction of $\vartheta$ depends upon the angle map, $\alpha$, which, in turn, requires the choice of a morphism, $F \in \mathcal{M}^{\omega}[T J, T J]$, whose symmetric part is positive definite. In the present case a convenient choice is specified as $F:\left(p_{1}, p_{2}\right) \rightarrow\left(p_{1}, K_{1}^{-1} p_{2}\right)$, for this leads to the simple angle map (??)

$$
\alpha \triangleq D \varphi F^{\top} M p_{2}=p_{1}^{\top} M p_{2}
$$

The coefficient functions, $\gamma_{i}$, may all be chosen constant as shown in $[4]$. The morphism, $B(x)$, is now computed as

$$
\begin{aligned}
\nabla_{x} F d \varphi & =\left(D_{p_{1}} F D \varphi\right) x+\Gamma(F D \varphi, x) \\
& =F D^{2} \varphi x+\dot{F}(D \varphi) x+\frac{1}{2} M^{-1}\left(M^{\prime}+J\right)(F D \varphi) x \\
& =I+0+\frac{1}{2} M^{-1}(M+J)\left(p_{1}\right) x
\end{aligned}
$$

and it follows that

$$
\mu_{(M B)},\left(\left\|p_{1}\right\|\right)=\mu_{M}\left(\left\|p_{1}\right\|\right)+\frac{1}{2} \mu_{(M)}\left(\left\|p_{1}\right\|\right) .
$$

Applying the assumption that $M$ and $D M^{s}$ have bounded magnitude on the configuration space yields the affine function

$$
v_{(M B)},(x) \triangleq \overline{\mu_{M}}+\frac{1}{2} \overline{\mu(M)} x \geq \mu_{(M B)}(x)
$$


hence

$$
\int_{0}^{x} v_{(M D)} \circ \lambda_{n}^{-1}(s) d s \leq \overline{\mu M} x+\frac{3 \overline{\sqrt{\mu(M)}}}{2 \sqrt{\overline{\nu_{M}}}} x^{3 / 2}
$$

The complete Lyapunov function is given by

$$
\vartheta \triangleq p^{\top} P_{p ;} \quad p \triangleq\left[\begin{array}{cc}
\left(\gamma_{0}+\gamma_{R} \eta^{1 / 2}\right) K_{1} & \frac{1}{2} M \\
\frac{1}{2} M & \left(\gamma_{0}+\gamma_{R} \eta^{1 / 2}\right) M
\end{array}\right]
$$

where the constant coefficients are defined in (4). Compare this to the total energy function,

$$
\eta \triangleq p^{\top} H_{p} \quad H \triangleq\left[\begin{array}{cc}
K_{1} & 0 \\
0 & M
\end{array}\right]
$$

\subsection{Input/Output Analysis of the PD Compen- sated System}

In this section we will refer extensively to the language and detailed computation in the technical report $[4]$ in order to extract explicit relationships between input and output bounds from the globally strict Lyapunov function, $v$.

Although it was shown in Theorem 1 that the construction has a negative definite derivative along the motions of the pd compensated unforced mechanical system (9), we are now interested in displaying an explicit quadratic lower comparison function for the specific case defined by (15).

Lemma 4 The negative derivative map, $-L_{f}(\vartheta)$, admits a quadratic lower comparison funclion,

$$
\lambda_{j}(x) \triangleq \nu_{Q} x^{2}
$$

where $Q$ is a positive definite symmetric matrix defined by the "feedback gains", $\Pi_{1}, K_{2}$, and "energy scaling constants", $\gamma_{P}, \gamma_{P}^{\prime}, \gamma_{Q}$, used in (15).

The lower comparison function, $\lambda_{j}$, may now be used to find a " $\beta$-descent region" (defined in $[4]$ ) - i.e. a region wherein the Lyapunov function, $\vartheta$, and, ultimately, the distance to the origin, along solutions to the forced system 0 is guaranteed to be decreasing as long as the forcing function is bounded by $\beta$.

Proposition 2 For every $\beta>0$ there exists a $\delta>0$ such that. if $\|u\| \leq \beta$ then $\dot{v}_{u}$ is strictly negative on the set

$$
P-P_{0}^{\delta} \triangleq\{p \in P:\|p\| \geq \delta\}
$$

This result leads immediately to the construction of a scent rate estimate" as defined in [4], which, in turn, demon. strates that $v$ is not only globally strict, with a radially un. bounded Lie derivative, but, when restricted to any compact domain, is uniform as well.

Proposition 3 For all $\chi_{1} \in \mathbb{R}^{+}, v$, as defined in Proposition 1 , is a uniform lyapunov function for $f$ on $D_{3} \triangleq p_{0}^{X_{1} / \sigma_{r}}$.

This yields the desired bound on the magnitude of the state of the forced system, expressed in terms of the "feedback gain" magnitudes and scaling coefficients.

Theorem 2 The motion of a PD compensated mechanical sys. tem (9) from the initial condition $\left\|p_{0}\right\|=\chi_{0}$, in response to inputs of magnitude less thon $\alpha<\infty$ is bounded by

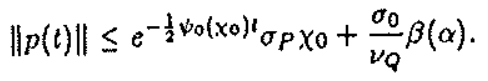

\section{References}

[1] Ralph Abraham and Jerrold E. Marsden. Foundations of Mechanics. Benjamin/Cummings, Reading, MA, 1978.

[2] S. Arimoto and F. Miyazaki. Asymptotic stability of feedback controls for robot manipulators. In Proceedings 1st IFAC Symposium on robot Control, Barcelona, Spain, 1085.

(3) Morris IV. Hirsch. Differential Topology. Springer-Verlag, : NY, 1976.

[4] D. E. Koditschek. A Sirict Global Lyapunov Function for Mechanical Systems. Technical Report 8707, Center for Systems Science, Yale University, November 1987.

[5] Daniel E. Koditschek. Adaptive tecliniques for mechanical systems. In Fifth Yale Workshop on Applications of Adaptive Systems Theory, page, Center for Systems Science, Yale University, New Haven, CT, May 1987.

[6] Dąniel E. Koditschek. Higlı gain feedback and telerobotic tracking. In Workshop on Space Telerobotics, page, Jet Propulsion Laboratory, California Institute of Technology, Pasadena, CA, Jan 1987.

(7) Daniel E. Koditschek. Natural motion for robot arms. In IEEE Proceedings 29rd Conference on Decision and Con. trol, pages 733-735, Las Vegas, Dec 1984.

(8) Daniel E. Koditschek. Robot control systems. In Stuart Shapiro, editor, Encyclopedia of Artificial Intelligence, pages 902-923, John Wiley and Sons, Inc., 1987.

(9) J. Milnor. Morse Theory. Princeton University Press, Princeton, NJ, 1963.

[10] Fumio Miyazaki and S. Arimoto. Sensory feedback based on the artificial potential for robots. In Proceedings oth IFAC, Budapest, Hungary, 1984.

[11] A. J. Van Der Schaft. Stabilization of Hamiltonian Systems. Memo 470, Technische Hogeschool Twente, Twente, Netherlands, Jan 1985.

(12) Jean-Jacques E. Slotine and Weiping Li. On the adaptive control of robot manipulators. In Proc. ASME Winter Annual Meeting, page, Anaheim, CA., Dec 1986.

(13) Morikazu Takegaki and Suguru Arimoto. A new feed. back method for dynamic control of manipulators. $A S M E$ Journal of Dynamics Systems, Measurement, and Control, 102:119-125, 1981 .

[14] Sir W. Thompson and P. G. Tait. Treatise on Natural Philosophy. University of Cambridge Press, 1886, Cam. bridge.

(15) John T. Wen and David S. Bayard. Robust Control for Robotic Monipulators Part I: Non-Adaptive Case. Technical Report 347-87-203, Jet Propulsion Laboratory, Pasadena, CA, 1987. 